\title{
LOS EJÉRCITOS ANTE LOS NUEVOS ESCENARIOS: RETOS Y ESPERANZAS
}

I Seminario franco-español de Sociología Militar, Toledo, 1-2 de Julio de 1999. Organizado por la Asociación Castellano Manchega de Sociología en colaboración con el Ministerio de Defensa.

Sumario:

Presentación: Primer seminario de sociología militar en Castilla-La Mancha. García Bilbao, P. A.; Martínez Paricio, J., A.C.M.S.

Sobre la evolución y reforma de las instituciones francesas de concertación profesional en las Fuerzas Armadas.

Rebmeister, C.G., Interventor General, Secretario general del Consejo Superior de la Función Militar.

Los militares franceses ante el actual sistema de concertación profesional: encuesta realizada por el Observatorio Social de la Defensa (1997).

Azoulay, P., Centre d'Études en Sciences Sociales de la Défense.

Libertad de expresión: uso y práctica.

Coronel de Richoufftz, Ejército de Tierra, Francia.

$* * *$

Presentación: Primer seminario de sociología militar en Castilla-La Mancha.

García Bilbao, P. A.; Martínez Paricio, J., A.C.M.S.

\section{I}

Cuando se vio que existía la posibilidad de organizar un primer seminario sobre cuestiones de sociología militar actual en Toledo, ciudad en la que impartiera su cátedra en la Academia Militar el general Vicente Rojo, modelo de militar profesional y demócrata, en la A.C.M.S. la asumimos con verdadero entusiasmo, empezando, sin duda, por nuestro presidente, el Dr. Uña Juárez. La creación de un grupo de investigación en el campo de la sociología militar formaba parte de los proyectos de trabajo trazados al poco de constituirse nuestra asociación. Aun a riesgo de corregir a Miguel Alonso Baquer, uno de los puntales de este campo, en la actualidad la sociología 
militar contemporánea en España centra la mayor parte de su esfuerzo investigador en tres tipos de problemas ${ }^{1}$ : los relacionados con la profesionalización de las Fuerzas Armadas y sus retos en el actual contexto mundial, los derivados del cambio de valores sociales y su impacto en la actitud hacia el reclutamiento y la defensa nacional y la carrera militar en sí misma (estudios sobre trayectorias profesionales, sistema de reproducción, etc.); todos ellos -y algún otro más- presentan un gran potencial temático para cualquier grupo de sociólogos que deseara aplicar a él su esfuerzo profesional.

Tras este seminario de Toledo, algunas de cuyas más sugerentes intervenciones aquí recogemos, la A.C.M.S. incluyó, en su congreso anual de Almagro, un panel específicamente dedicado a la sociología militar que esperamos tenga continuación en convocatorias venideras. Desde estas sencillas líneas de presentación quisiéramos animar a nuestros colegas profesionales (sociólogos y politólogos) a contactar con el grupo de trabajo de la Asociación (soc.militar@acms.es); nuestro planteamiento es sencillo: plena libertad de actuación y absoluto rigor académico como únicos límites deseables. Las intervenciones que se exponen en este informe especial han sido tomadas de las cintas que siguieron el seminario, traducidas y transcritas en origen; las versiones que se presentan han sido objeto de algunas leves correcciones de estilo y de las que es único responsable quien esto suscribe. (Pedro A. García Bilbao).

II

El fin de los bloques arrumbó los elegantes modelos que explicaban las relaciones internacionales. La historia no ha terminado. Es más, los acontecimientos que vivimos indican que la historia marcha por delante de los hombres.

Los ejércitos de las sociedades modernas se encuentran inmersos en un complejo proceso de cambio que, al tiempo, se produce dentro de un cambio más amplio y transcendental.

Como se anticipó hace tiempo, corrían los años sesenta, los ejércitos del «futuro» tendrían que hacer frente a distintos retos. La organización tendría que integrar en la misma estructura distintas funciones: las clásicas y otras que comenzaron a denominarse de manera poco precisa como «nuevas», y debería hacerlo en un organigrama donde la reducción de plantillas sería un principio fundamental. Por otro lado, la doctrina que regulaba el empleo de los recursos materiales y humanos se mostró insuficiente. El ejército, como organización institucional, debería integrar la tradición con la modernidad. Se avisó que los ejércitos nacionales tendrían que integrarse en organizaciones supranacionales, con los problemas simbólicos y de identificación que esto acarrearía.

Pocas veces las anticipaciones sociológicas estuvieron tan medidas.

Para llevar a cabo todos estos procesos de adaptación, que no son los únicos, los gestores de los asuntos militares tendrán que tener presente que los recursos económicos con los que podrán contar serán cada vez más reducidos y con ellos tendrán que hacer frente a las exigencias crecientes del nuevo ejército. 
Un problema no menos complejo tiene que ver con el papel del ejército en la sociedad venidera, nacional e internacional. Los aspectos de la seguridad y la defensa deberán incorporarse a la cultura cívica propia de ciudadanos.

El sistema de relaciones de los ejércitos y de los militares en las sociedades modernas y avanzadas, se tendrán que plantear bajo otros supuestos. Pero quizás el aspecto no menos central del cambio va a ser, está siendo ya, el nuevo concepto que tiene el militar profesional de sí mismo y de su oficio.

No resulta aventurado afirmar que los ejércitos y los militares de ahora y del futuro tendrán que acostumbrarse a vivir en una situación de crisis de identidad importante. Es más, tendrán que saber gestionar esa crisis.

Estos asuntos son los que motivaron la reunión de Toledo. Todo lo anterior, que no es nada más que una parte del problema global al que se están enfrentando los ejércitos de las sociedades modernas, es motivo más que suficiente para reflexionar de manera abierta, sosegada y sin prejuicios. Incluso hay que reconocer que es una obligación de la que no se puede hacer dejación. La seguridad es cuestión de todos. Que todos se interesen por ella, y con la misma intensidad, es otra cuestión. En estas circunstancias y en estas materias, la reflexión y la crítica fundada son imprescindibles.

La Asociación Castellano-Manchega de Sociología (A.C.M.S.) promovió con la colaboración de la Universidad de Castilla-La Mancha- el encuentro. Desde aquí agradezco la buena disposición de todos sus miembros, en concreto los de su Ejecutiva. A la reunión se sumaron alumnos y colaboradores de la Facultad de CC. PP. y Sociología de la Universidad Complutense, de la de Granada, Deusto, La Coruña y Barcelona. Un nuevo motivo de satisfacción, pues las envidias, celos y recelos dejaron paso a la reflexión conjunta.

El encuentro se celebró los días, 1 y 2 de Julio de 1999. El tema estrella tratado, de gran importancia y transcendencia política, fue La libertad de expresión entre los militares. Se pudo comprobar que no es un mito. Nadie puso ningún obstáculo y la claridad y apertura de ideas llenó el salón de la Inquisición toledana.

El encuentro contó con el apoyo incondicional del Instituto Español de Estudios Estratégicos. Aprovecho la ocasión para agradecer el apoyo de su Secretario General Permanente, almirante Lapique y todo su equipo con el coronel Capel a su cabeza.

Además de expertos, profesores y alumnos, participaron colegas franceses que debaten sobre las mismas cuestiones y en los mismos términos. Se trató de una representación del Centre d'Études en Sciences Sociales de la Défense (C2SD), organismo oficial encargado de analizar y promover el estudio comparado de estas materias, desde y en la universidad francesa, y en colaboración con universidades de países vecinos. Esta reunión es un buen ejemplo. Junto con personalidades relevantes del Centro, participarán otros profesores, del Centre d'Études et Recherches sur l'Armée, de la Universidad de Toulouse, así como militares y responsables de la administración militar francesa. Acudieron un número importante de miembros del Centro, su director: Contrôleur Géneral Hoffmann, C.G. Rebmeister, secretario del Consejo Superior de la 
Función Militar y asesor del Ministro de defensa de Francia, el teniente coronel Guillamó, (doctor en sociología por la Sorbona y Master en relaciones Internacionales), enlace de la Agregaduría de Defensa en Madrid que, junto a Bernard Labatut, profesor y buen conocedor de España, actuaron además de sufridos traductores. En esta labor impagable actuó también Ana Roel, imprescindible su tarea.

Por parte española citar algunas personalidades. El anterior Jefe del Estado Mayor de la Defensa, teniente general Faura, el también teniente general Chamorro, que fue Jefe del Mando Aéreo de Combate, doctorando en la Complutense, Vicente Lanz, del Gabinete Jurídico del Ejército del Aire, o el Sr. García Rodríguez, doctor en Cc. Políticas por la Complutense y antiguo director de la Academia General del Ejército del Aire.

Al margen de cualquier otra idea, los temas son de gran interés para un grupo de profesionales que reflexiona sobre el vivir de nuestra sociedad, de las condiciones políticas en las que nos desenvolvemos, de cómo cambian las organizaciones complejas, cómo se adaptan las instituciones a los nuevos escenarios, qué efectos tiene sobre las profesiones... Son reflexiones que, a parte de la militar, puede ser de interés para los miembros de la Asociación.

Motivo de satisfacción, insisto en el agradecimiento, es que en Almagro se organizó un panel sobre estas materias. Es un principio que cabe esperar sea el primer mojón de un largo camino. Resultado de estos éxitos acumulados es el curso que se dictará en los próximos meses organizado esta vez en el ámbito de reflexión de la Universidad Rey Juan Carlos. Aquí también el Instituto echará su cuarto a espadas para que se pueda llevar a buen puerto esta experiencia novedosa en la Universidad española. Es hora ya que estas materias, imprescindibles en una sociedad moderna, sean estudiadas por los universitarios. (Jesús Martínez Paricio).

Sobre la evolución y reforma de las instituciones francesas de concertación profesional en las Fuerzas Armadas.

C.G. Rebmeister, Interventor General, Secretario General del Consejo Superior de la Función Militar² . Antes de empezar, quisiera expresarles la gran satisfacción que supone para mí estar junto a ustedes en Toledo y ello por muchas razones.

Primero, porque siempre es agradable hablar de lo que se hace, cuando se tiene la suerte de amar aquello a lo que uno se dedica. Ejerzo, en Francia, la función de Secretario General del Consejo Superior de la Función Militar (S.C.F.M.), es decir, de la instancia nacional de concertación de los militares franceses. Siempre es para mi una gran satisfacción poder hablar de esta función, un poco especial, que desempeño en el seno del dispositivo de defensa francés.

En segundo lugar, porque siempre es agradable hablar en un ambiente tan simpático, en medio de una audiencia tan interesada y competente sobre el tema. 
Finalmente, porque, como bien saben. Los militares de los diferentes países de la unión europea aprendieron, desde hace algunos años, a trabajar juntos por tierra, mar y aire. Los acontecimientos actuales hacen que esta tendencia marcará cada vez más la pauta y, ni que decir tiene, repercutirá necesariamente sobre las diferentes modalidades de derecho de expresión individual de los mismos, pero también sobre su derecho de expresión colectivo.

Por esta razón, es especialmente grato para mi poder ofrecerles hoy a la vez el balance de nuestro dispositivo tal como funciona en la actualidad y también nuestros interrogantes en un momento en que intentamos modificar dicho dispositivo, ante ustedes, en un país amigo de la unión europea, cuyos militares están llamados a codearse cada vez más con nuestros militares franceses.

Unas pocas palabras para indicarles el plan según el cual me propongo abordar el tema que se me pidió que tratara, es decir las instancias de concertación de los militares franceses en la actualidad. Mi intención es hacerlo en cuatro puntos, tratando de contestar a cuatro preguntas.

¿Por qué unas instancias de concertación para los militares franceses, $y$ desde cuándo?

¿Cuáles son actualmente dichas instancias de concertación a disposición de los militares franceses'?

¿Por qué las modificamos ahora, y en qué sentido queremos hacerlo?

Por último, pretendo hacer ante ustedes algunas reflexiones en voz alta sobre la problemática de la concertación en el Ejército y, más especialmente, en el Ejército francés (... $)^{3}$

(...) Quedémonos, pues, para simplificar, con que los militares franceses [en relación a la primera cuestión] disponen de instancias de concertación desde principios de los años 70 y que, en su gran mayoría, la decisión de institucionalizar dichas instancias de concertación se adoptó a raíz de acontecimientos sociales, tales como el mayo del 68, o de acontecimientos más específicos de la colectividad militar, como los «comités de soldados» con el fin de evitar que los mandos militares o los jóvenes reenganchados no se vieran, a su vez, contaminados por ese virus contestatario que caracterizó los años 70.

¿Cuáles son las instancias de concertación de qué disponen actualmente los militares franceses?

Para describir de la forma más sencilla y esquemática posible el dispositivo se puede decir (...) que existen instancias de concertación a dos niveles principales: las instancias nacionales y las instancias locales Veremos más adelante que pretendemos implantar algo a nivel intermedio, el nivel regional, pero por ahora no es posible.

1) Ámbito local.

¿Qué tenemos en este ámbito? : Presidentes de categoría y comisiones de participación de las unidades... 
- Presidentes de categoría.

Estos presidentes de categoría podemos encontrarlos en casi todos los ejércitos o servicios, de acuerdo con diferentes modalidades que varían de un ejército a otro o de un servicio u otro. En general, podemos decir que se cuenta con toda seguridad... :

a) ... casi en todas partes, con presidentes de suboficiales,

b) ...casi en todas partes, con presidentes de oficiales siguiendo diferentes modalidades según los ejércitos (algunos ejércitos disponen de un único presidente de oficiales; otros tienen un presidente de oficiales subalternos y un presidente de oficiales superiores; otros no tienen ninguno),

c) ...en los ejércitos que cuentan militares de reemplazo, un presidente de soldados de reemplazo, cuya denominación varía de un ejército a otro.

Por tanto y por regla general, y cuando el sistema está al completo, existen uno o varios presidentes de oficiales, uno o varios presidentes de suboficiales y un presidente para los soldados de reemplazo. En todos los casos, excepto en la gendarmería, estos presidentes son designados por el mando del regimiento, del buque o de la base aérea a propuesta del presidente saliente, que generalmente se presenta a su jefe con una lista de dos nombres, elegidos por orden por sus iguales. El jefe nombra a un presidente que suele ser, en el noventa por ciento de los casos, el primero de la lista que se le entregó.

Por el contrario, en la gendarmería, y ello desde hace aproximadamente diez años, el presidente de los suboficiales es designado por sus iguales, lo cual resulta ser una expresión púdica, por no decir que es elegido por sus compañeros.

¿Cuáles son las funciones de estos presidentes?

Son, fundamentalmente, funciones de representación y de defensa de los intereses de la colectividad a la que representan, la categoría de militares a los que representan. Son asimismo, en cierto modo, asesores de la autoridad militar a nivel regimiento, buque o base aérea. Cada día más, en ciertos ejércitos desempeñan igualmente un papel hacia el exterior, y no es extraño ver, en calidad de invitados a una ceremonia militar o a una manifestación patriótica, no sólo al jefe del regimiento, sino también a los presidentes de oficiales, de los suboficiales o del personal de tropa.

Éste es el primer nivel de representación, una vez más, con diferentes modalidades que pueden variar de un ejército a otro, pero en general y salvo casos concretos, cada vez que los efectivos militares implantados en un lugar dado -constituyendo una formación-, sean suficientes, habrá presidentes y, en todo caso, casi en todas partes, un presidente de suboficiales y un presidente de soldados, Llegado el caso.

- Comisiones de participación.

Junto a estos presidentes de categoría se encuentra una auténtica instancia de concertación, y ya no un individuo o un grupo de jindividuos: una comisión, 
cuyo apelativo global para defensa es "Comisión de participación de la unidad», pero puede tener otras denominaciones según los diferentes ejércitos. En todos los casos se trata de una comisión en la cual volvemos a encontrar representantes de la autoridad militar - generalmente el jefe del regimiento, buque o base aérea, algunos de sus colaboradores principales, los presidentes de las categoríasque mencioné anteriormente - los miembros de las instancias nacionales — a los que aludiré más adelante- cuando existan en la unidad, y militares elegidos para representar a las diferentes categorías de personal. Estas comisiones, a diferencia de las instancias nacionales, no tienen por misión pronunciarse sobre los grandes temas relativos a la condición y al estatuto de los militares actualmente. Su función consiste, esencialmente, en resolver problemas concretos, locales, de funcionamiento de la vida diaria, ya se trate de problemas ligados a la circulación, al estacionamiento, al horario de trabajo, a la alimentación o al tiempo de ocio. En resumen: todo lo relacionado con la vida diaria que, generalmente, sólo puede solucionarse a nivel local, y cuya resolución depende, generalmente, del presidente de la comisión y sin que, por ahora, dichos trabajos puedan repercutir en los problemas estatutarios generales relativos a la condición o al estatuto de los militares. Ámbito nacional.

En lo que se refiere al nivel nacional, vamos a encontrar ocho consejos: siete consejos para la función militar y un consejo superior de la función militar.

- Siete consejos de la función militar. A saber:

el ejército de tierra,

la marina,

el ejército del aire,

la gendarmería,

la delegación general de armamento,

el servicio de sanidad del Ejército,

el servicio de carburantes del Ejército.

- Un consejo superior de la función militar que representa a la totalidad de estos siete componentes de la defensa.

Estos consejos, que son inseparables, los siete consejos y el consejo superior, son la culminación de un proceso que se inició en el año 1969 con la creación de un consejo único superior y se fue enriqueciendo con el paso de los años con la creación, en 1990, de los siete consejos de la función militar. Estamos reflexionando actualmente sobre las mejoras que podrían aportarse a este dispositivo.

¿Cómo funcionan estos consejos? Están compuestos por militares en activo, elegidos por sorteo entre los voluntarios y únicamente por el consejo superior, la instancia interejércitos, completado con seis militares en la reserva, los cuales representan a las seis asociaciones más representativas del personal en la reserva.

En los siete consejos de los ejércitos, únicamente militares en activo; en la instancia nacional interejércitos: una representación de los militares en la reserva. 
Estos militares son, por tanto, todos ellos voluntarios y participan en la candidatura. Se eligen por sorteo por un periodo de cuatro años. La mitad del consejo se renueva cada dos años.

Sus miembros disponen de garantías...:

- deben gozar del tiempo necesario para la preparación del tema de estudio antes de las sesiones,

- tienen derecho a expresarse con toda libertad durante los trabajos del consejo,

- en la hipótesis de que fueran objeto de presiones, pudieran sufrir retrasos en su carrera o perjuicios de cualquier otro tipo por su pertenencia al consejo gozan de un derecho de recurso específico, ya que pueden apelar directamente a mí de tal modo que yo pueda asegurarme de los hechos por los que pudiera guardárseles rencor debido a su participación en el consejo.

Estos consejos trabajan casi todos de la misma forma: los siete consejos de la función militar preparan los trabajos, y el consejo superior los culmina y resume.

Las reglas que voy a mencionar son válidas. excepto algunos casos, para la totalidad de estas instancias.

El orden del día de estas instancias lo decreta el Ministro, ya se trate del consejo superior o de los siete consejos, con la posibilidad de inscribir de oficio una cuestión, si un tercio de los miembros como mínimo lo solicita. En realidad, la cosa es mucho más flexible que todo esto, y no se conocen antecedentes desde hace 30 años que el sistema existe, en que el gabinete del Ministro haya rechazado la inscripción de un tema en el orden del día. Nunca hubo necesidad de utilizar esta posibilidad teórica de solicitud de inscripción de oficio de una determinada cuestión.

Cada uno de los consejos celebra dos sesiones ordinarias anuales, de una semana de duración aproximadamente: una, denominada «de primavera», que tiene lugar a finales del mes de mayo, la otra denominada "de otoño», que se celebra a finales del mes de noviembre o a principios del mes de diciembre.

Estas sesiones se clausuran en lo que se refiere a los siete consejos del ejército a veces por el Ministro, pero con toda seguridad por los jefes de Estado Mayor o por los directores generales; en el caso del Consejo Superior, de forma sistemática por el Ministro en presencia de las más altas autoridades militares y civiles del Ministerio.

Estas dos sesiones se articulan, en líneas generales, en cinco tiempos:

- Los consejos empiezan haciendo un balance del curso que se dio a los trabajos de las sesiones precedentes. ya se trate de proyectos de textos sobre los que formularon sugerencias o temas de estudio.

- A continuación, analizan un cierto número de proyectos de textos legislativos o reglamentarios referidos a la condición o al estatuto del personal militar. Formulan un dictamen que, para los textos más importantes relativos a los estatutos particulares de los militares, se comunica al Consejo de Estado cuando éste examine el proyecto del decreto. 
- En un tercer tiempo, estudian un tema general. Este año, por ejemplo, los consejos sugieren las consecuencias prácticas, en términos de condición militar, de la profesionalización del Ejército.

- El cuarto tiempo lo constituye la sesión plenaria del consejo que se celebra en presencia de las más altas autoridades civiles y militares del Ministerio en el caso del Consejo Superior y de las más altas autoridades de cada ejército, dirección o servicio para el caso de los siete consejos.

- Le siguen, por último, una o varias sesiones de información que son información interna en las que los miembros [establecen un diálogo directo] sin ningún «lenguaje ritual»-como se dice en Francia - por parte de las autoridades civiles o militares del Ministerio o sesiones de información exterior para las cuales puedo requerir a personalidades ajenas al Ministerio, procedentes de otros Ministerios, e incluso del mundo de las sociedades o mutualidades, para que vengan a difundir información sobre temas de interés para el consejo. A título de ejemplo, el consejo había solicitado que se informase acerca de la evolución del S.I.D.A. en el Ejército francés. Más recientemente, asociaciones de esposas de militares vinieron a presentarnos lo que estaban haciendo en beneficio de la colectividad militar.

Los trabajos de estos consejos son objeto de gran publicidad. Cada uno de los consejos publica al término de su sesión plenaria [diversos documentos].

Un comunicado, en el que se recoge su punto de vista, y se difunde en forma de mensaje a la totalidad de las unidades del ejército considerado, por los consejos de ejército, y a todas las unidades de defensa, por el consejo superior.

Un informe, -este documento más espeso-, que consta de 100 a 150 páginas en el caso del Consejo Superior, se difunde con algunas semanas de diferenciacuando no algunos meses - por razones de impresión. Desde hace algunos meses, y por decisión del Sr. Richard, actual Ministro de Defensa francés, las revistas de prensa escrita militar remiten lo más pronto posible después de cada sesión, una versión resumida de dichos informes, de tal modo que estén rápida y fácilmente accesibles al mayor número posible de militares.

¿Por qué modificamos actualmente este dispositivo y en qué sentido pretendemos hacerlo?

En primer lugar... ¿por qué iniciar en 1997, con la llegada del Sr. Richard al frente del Ministerio, una reforma de las instancias de concertación de los militares? Fundamentalmente por dos razones:

Primera, adaptar el dispositivo actual a un ejército profesional.

Como les he dicho, habíamos implantado a nivel local unas comisiones de participación orientadas fundamentalmente a los soldados de reemplazo. y a nivel nacional unas instancias de concertación orientadas más especialmente a los mandos. En el ejército profesional del 2002, y desde ahora en el ejército mixto actual en el que el número de soldados reenganchados es cada vez mayor, el 
personal de tropa se convertirá ya en gran medida en personal profesional. No podíamos pasar por alto un cambio tan importante dentro de la organización del dispositivo de concertación. En otras palabras, en un ejército de reclutamiento en el que los soldados, que son fundamentalmente soldados de reemplazo y sólo permanecían un corto periodo de tiempo en el mismo, podían contentarse con unas instancias locales que tratasen únicamente problemas locales. En un ejército profesional, estos soldados de reemplazo serán voluntarios con contrato y estarán un tiempo más o menos largo dentro del ejército. Es normal que tengan un mayor peso en las instancias nacionales, las cuales abordan cuestiones relacionadas con la condición y el estatuto de los militares.

Segunda. Continuidad de una evolución iniciada en 1969.

El C.S.F.M. se creó en 1969. El dispositivo experimentó una importante reforma en 1990 y, actualmente, unos diez años más tarde, tenemos la sensación de que hay que dar un segundo respiro a esta reforma a fin de poder tener en cuenta la evolución de las mentalidades. Podemos alegrarnos o lamentarlo; pero en todos los ejércitos del mundo, los militares no son, finalmente, más que antiguos civiles convertidos en militares y con cierta inclinación a volver a convertirse en civiles. Quiero decir con esto que no es nada extraño que nuestros militares lleguen al Ejército siendo portadores de los valores, la cultura y la sensibilidad de una sociedad civil en evolución. La sociedad militar, por tanto. evoluciona ella también, y es normal que las instancias de concertación también evolucionen.

Existe una tercera razón, un poco menos confesable. Es el afán por no querer dejarse superar por los acontecimientos y mejorar nuestros sistemas tomándonos el tiempo necesario para hacerlo con serenidad antes que en caliente. En 1990, el Ministro de Defensa en aquél entonces, el Sr. Chevènement, tuvo que reorganizar a toda prisa las instancias de concertación tras una crisis que habla convulsionado la gendarmería y que se tradujo, durante el verano de 1989, en el envío de un cierto número de cartas anónimas denunciando las dificultades por las que pasaban los gendarmes.

Son tres las razones, pues, que nos empujan a llevar a cabo la reforma: [primera] tener en cuenta la profesionalización, [segunda] proseguir con la reforma iniciada hace 10 años y que empieza a perder un poco de fuelle $y$, finalmente, [tercera] adelantarse a los acontecimientos a fin de no correr el riesgo, algún día, de tener que modificar a toda prisa nuestro dispositivo para tener en cuenta cualquier atisbo de malestar que pudiera presentarse.

[Modificaciones] a nivel nacional:

Paso por alto la metodología que hemos utilizado, que no es demasiado espectacular, a no ser porque ha sido objeto de una larguísima concertación -es lo menos que puede hacerse en nuestro ámbito cuando se acomete una reforma- para pasar en seguida a las ideas principales que sirven de base a esta reforma o, más exactamente, a lo que será esta reforma 
No cuestionábamos de manera trascendental la economía general de las instancias nacionales, es decir, que no existen turbulencias en las instancias nacionales que acabo de reseñarles. Pero aportamos a las mismas una serie de innovaciones de carácter técnico cada una de las cuales, tomada por separado, puede parecer poco importante, pero que estoy convencido mejorarán la representatividad (...) y proporcionarán una mayor eficacia a los consejos. Adaptamos la composición de los consejos a la futura fisionomía de los ejércitos. aumentando el peso relativo del personal de tropa

Tenemos más en cuenta, asimismo, la diferenciación entre militares de carrera y militares con contrato, ya que en el Ejército profesional del mañana en Francia, uno al menos de cada dos militares será militar con contrato. Mejoramos el dispositivo de suplencia. Es un poco técnico pero en definitiva quiere decir que habrá menos suplentes, pero que tendrán una mayor formación y que podrán sustituir mejor a sus compañeros titulares cuando éstos no puedan celebrar sus reuniones. Introducimos. siempre que ello sea posible, un criterio geográfico en el sorteo, de tal modo que haya, en la medida de lo posible, una distribución lo más justa posible de miembros por todo el territorio nacional, al objeto de que puedan recoger mejor los deseos de sus compañeros y puedan informar mejor hacia los escalones más bajos. Extendemos y armonizamos la formación a los nuevos miembros a fin de que puedan desempeñar más eficazmente su cometido. Esto a nivel nacional.

[Modificaciones] Nivel regional:

A nivel regional, implantamos, cada vez que ello parezca justificado, una instancias intermedias o regionales de enlace entre las instancias nacionales (los consejos) y las instancias locales (los presidentes de categoría y las comisiones participantes) -y esto será así, principalmente, en el ejército de tierra, en la marina y en la gendarmería. No se trata de crear consejos a nivel regional. Lo hemos intentado, pero no dio buenos resultados. Se trata de actuar de tal modo que los miembros de las instancias nacionales, antes de reunirse en París, o después de haberlo hecho, puedan recoger mejor las preocupaciones de los militares de las instancias locales y puedan explicar mejor sus trabajos, las acciones que hayan llevado a cabo y las notificaciones que se les hayan hecho. Esperamos, pues, de las instancias regionales una mejor circulación de la información.

[Modificaciones] a nivel local:

[En lo local], algunas modificaciones no exentas de consecuencias. Primero, informamos de la existencia en cada formación, cualquiera que sea su importancia, de un representante de los consejos al que podrá consultarse para recabar documentación, para conocer los comunicados, el cual podrá trasladar la información hacia los escalones superiores. Pedimos que se valore y confirme mejor el papel de los presidentes de categoría allí dónde menos lo estaba, es decir, especialmente entre el personal de tropa. 
Ampliamos la competencia de las comisiones participantes de las unidades, en el sentido de que puedan no sólo tratar problemas locales relativos a la vida diaria, sino asimismo recoger información hacia arriba y difundir la información resultante de los trabajos de los consejos.

Finalmente, y es ésta sin duda alguna la parte más espectacular de la reforma, nos preguntamos sobre la conveniencia de extender a la totalidad del Ejército, Direcciones y Servicios lo que ya está haciendo la gendarmería - como ya les he señalado-, es decir, que la elección de los presidentes de categoría no corra ya a cargo del mando, sino de sus iguales.

Sobre este último punto, el poder político no tomó ninguna decisión, pero el Consejo Superior de la función militar remitió un comunicado en este sentido, en el transcurso de la sesión de otoño de 1998. No puede descartarse totalmente que en estos momentos se esté caminando en esa dirección, dado que el Ministro de Defensa, que se reunió para hablar de ello con las más altas instancias del Estado, no dio todavía a conocer su postura sobre este punto.

Para concluir, y rápidamente, desearía hacerles partícipes de algunas reflexiones en voz alta acerca de estos problemas generales de concertación en los ejércitos.

Estas reflexiones son un poco el fruto de dos años de experiencia al frente del Consejo Superior, [y] sobre todo, de dos años de trabajo sobre la reforma de las instancias de concertación, durante los cuales trabajé duro. reflexioné e intercambié ideas.

El primer punto, ante todo, es que cuando se implantan, como lo están haciendo ustedes, instancias de concertación, o cuando se reforman dichas instancias tal como estamos haciendo nosotros, hay que tener presente que. como se suele de ir en Francia, «lo mejor puede ser enemigo de lo bueno». Quiero decir con ello que queriendo perfeccionar una cosa a veces se corre el riesgo de estropearla. Con el pretexto de conseguir unas instancias altamente eficaces, hay que abstenerse de implantar un dispositivo tan pesado y apremiante para los miembros de las instancias que pudiera materializarse en algo que nadie desea, es decir, que los militares en campaña, los militares de las formaciones operativas que constituyen las fuerzas vivas de los ejércitos, se alejen de dichas instancias dado que participar en las mismas exija demasiado tiempo y que los consejos queden finalmente compuestos por personal que desempeñe unas funciones un poco secundarias dentro del Ejército y que, en otras palabras, dispone de un poco más de tiempo que el resto para encargarse de todo ello. Esto sería algo totalmente dramático. Hay que estar, pues, alerta. Creo que lo realmente importante es que se permita reunirse a gente procedente de unidades operativas, que son quienes viven día a día los verdaderos problemas que se plantean en el Ejército. Si queremos contar con esta gente hay que encontrar el justo término medio entre el tiempo necesario para celebrar las sesiones de forma útil e inteligente y el tiempo más allá del cual desistirían de asistir como voluntarios. 
El segundo punto es que resulta muy importante que la gente goce de una auténtica libertad de expresión, y ello no solamente en los textos. Esto quiere decir que, sobre todo en las sesiones, hay que actuar efectivamente de modo que, sea mediante pequeños detalles de organización o mediante la forma en cómo transcurren los debates, tengan ciertamente el sentimiento de que no están controlados; con gente evidentemente para alentarlos, pero que tengan el sentimiento claro de que se hace la distinción entre los periodos de información, los periodos de restitución de trabajos, en los se requiere la presencia de un máximo de autoridades, y los periodos de deliberación en los que los miembros deben estar a solas, a puerta cerrada, sin nadie de afuera. La gente encargada de ayudarles, en nuestra organización los secretarios generales -como es mi caso para el Consejo Superior- sólo interviene, de hecho, para ratificar sus resoluciones a fin de que sean perfectamente comprensibles para las autoridades, pero sin que por ello tengan la impresión de que la gente les observa para informar "a no sé quién», en particular a su jerarquía, de los puntos de vista que pudieron defender tal o cual persona. Nada habría más lamentable que oír al término de una sesión "iVaya!, el capitán Fulano de Tal parecía especialmente virulento». Creo que esto mataría la confianza que los miembros otorgan a las instituciones.

El tercer punto está relacionado con el segundo y se refiere al estatuto de la gente encargada de animar los trabajos. En nuestro sistema, los secretarios generales de los siete consejos son oficiales del Ejército, el secretario general del consejo superior es un interventor del Ejército, al igual que yo, es decir, alguien perteneciente a un cuerpo especial no dependiente del Ministro, libre de jerarquía. Soy Interventor General, tengo 47 años, estoy en mi último grado. En el peor de los casos, si llegase a desagradar a la autoridad política, volvería a mi Cuerpo y desempeñaría las misiones propias de la Intervención. Pero, para ser sincero, e incluso trivial, debo decir que no perdería un céntimo de mi sueldo a final de mes. Todo esto es importante, porque ello me confiere una tremenda independencia. No conozco exactamente los estatutos y las reglas por los que ustedes se rigen. pero creo que es preciso que tengan muy en cuenta, como nosotros tenemos, que es importante que los animadores de las instancias de concertación estén sujetos lo menos posible a eventuales presiones.

El cuarto punto, igualmente importante, es que está bien pedir a la gente que se exprese, permitirles hacerlo con toda libertad, asegurarse de que sus carreras no se verán afectadas, pero está todavía mejor tener en cuenta lo que dicen. En otras palabras, no puede concebirse que instancias serias, con unos miembros altamente cualificados, muy representativos, que trabajan bien, se limiten a un simple papel de válvulas de seguridad y que puedan aceptar que se les pida su opinión, sin tenerla nunca en cuenta y simplemente dejarlos liberarse. Si fuera así, creo que rápidamente lo entenderían y que, muy pronto, nos quedaríamos sin voluntarios cualificados. 
El último punto, y creo que todos nosotros nos hallamos impotentes ante tal evidencia, es que la eficacia de las instancias de concertación para los militares está bastante ligada a la personalidad de los jefes militares del momento y del Ministro del momento. Cualesquiera que sean los textos, por muy bien hechos que estén, si los Jefes de Estado Mayor, si los directores centrales de los servicios, no creen en la concertación, las instancias no servirán para nada.

En el ámbito del Ministerio, si el Ministro no cree en las instancias de concertación, si no acepta el juego del diálogo directo con los miembros, si los miembros no tienen la sensación de que está contento cuando está con ellos, que tiene en cuenta sus opiniones, que los respeta, entonces las más bellas construcciones intelectuales no servirán para nada.

Esto es todo lo que tenía que decirles. Espero no haberme extendido demasiado. Soy muy consciente del hecho de que todo lo que hemos construido en Francia no es forzosamente extrapolable a España, ni siquiera a otros lugares. Espero, no obstante, haberles aportado un testimonio lo más honrado posible del oficio apasionante que desarrollo día a día en mi papel de animador de estas instancias de concertación, tratando de hacerlas más eficaces. Ahora, estoy por supuesto a su entera disposición para contestar a las preguntas que ustedes quieran hacerme sobre lo que acabo de decir, sobre lo que no he dicho y, en definitiva, sobre el mundo de la concertación en los ejércitos.

C. G. Rebmeister

Los militares franceses ante el actual sistema de concertación profesional: encuesta $^{5}$ realizada por el Observatorio Social de la Defensa (1997).

Azoulay, P., Centre d’Études en Sciences Sociales de la Défense.

A finales de 1996, el Ministro de Defensa [francés] solicitó la elaboración de una encuesta de consulta en el Ejército.

Primero- Objetivos de la encuesta

Los dos objetivos principales perseguidos en la encuesta eran:

evaluar la adecuación del sistema a las necesidades militares.

conocer las expectativas y las evoluciones ansiadas por los militares.

Segundo- Metodología.

La encuesta se realizó en dos fases sucesivas. Primero a partir de un enfoque cualitativo y, después, un enfoque cuantitativo que aprovechó los resultados del primero (principalmente en lo tocante a la definición del cuestionario).

La encuesta cualitativa fue dirigida por la Sra. Léon, socióloga. Se llevaron a cabo un centenar de entrevistas. Éstas permitieron determinar los conocimientos de los militares acerca de las instancias de consulta así como conocer algunas de sus expectativas. El reducido número de entrevistas hace que los resultados obtenidos en esta encuesta cualitativa no puedan generalizarse. Es por ello por 
lo que se llevó a cabo una encuesta cuantitativa.

La encuesta cuantitativa fue realizada por el Observatorio Social de Defensa, a partir de un cuestionario elaborado en cooperación con los Secretarios Generales del C.S.F.M y de los C.F.M; se estableció contacto con 4.264 militares, contestando 1.748. La tasa de respuesta alcanzada fue del $40 \%$, cifra que puede considerarse elevada para un periodo de encuesta veraniego (en julio-agosto 1997).

La muestra inicial, representativa de la población militar.

La configuración de una muestra inicial representativa de la población militar se concretó a partir de datos estadísticos extraídos de los ficheros «retribuciones» de la Dirección de Personal.

Con este fin, se utilizaron tres variables:

arma o cuerpo,

rango militar.

antigüedad.

La muestra de los que contestaron.

La muestra de los que contestaron fue objeto de un control adicional. Se utilizaron tres variables:

edad.

estado civil.

lugar de destino.

El control de las seis variables anteriores permite asegurarse de que la estructura de la muestra de los que contestaron es similar a la de la población matriz, a excepción, sin embargo, de una sobrepresentación de soldados de reemplazo $\mathrm{y}$ de militares solteros.

Tercero- El sistema de consulta es aceptado de buen grado y sus trabajos se juzgan globalmente eficaces.

- La notoriedad de las instancias nacionales [de concertación].

El índice de notoriedad corresponde a la proporción de personas que conocen una institución, una marca, etc. El del C.S.F.M. es del $85 \%$, y del $77 \%$ para el CFM.

Estos índices varían de un ejército a otro: son más altos en la gendarmería (respectivamente, del 93\% y del $86 \%$ para el C.S.F.M. y el CFM), siendo más bajos en el Ejército de Tierra (79\% y $67 \%)$.

El indicador de notoriedad es mayor entre los oficiales y los suboficiales superiores (más del 95\% para el C.S.F.M. y alrededor del $90 \%$ para el CFM). Es menor entre los soldados de reemplazo (respectivamente, $36 \%$ y $32 \%$ ). Esta diferencia se debe a la menor edad y al menor tiempo de servicio de estos últimos.

- El conocimiento real.

La evaluación del conocimiento real se estimó a partir del:

- conocimiento de la posibilidad que todo militar tiene para preguntar directamente al C.S.F.M. y al CFM;

- conocimiento de las acciones del C.S.F.M. y de los CFM en lo que se refiere a 
las modificaciones del sistema de limite de edad, las nuevas ayudas para la readaptación, las nuevas ayudas a la hora de las licencias o también el quinto nivel de los capitanes.

Un poco más de un tercio de los militares encuestados (38\%) conocen la posibilidad de poder preguntar directamente a las instancias nacionales de consulta. Este porcentaje es muy antagónico: es bajo en los soldados de reemplazo (13\%) y bastante alto entre los oficiales (63\%).

El conocimiento de las actuaciones del C.S.F.M. respecto a las modificaciones del límite de edad es mayor en el Ejército del Aire dónde el 61\%de los que contestaron respondieron afirmativamente: en el Ejército de Tierra alcanza el $47 \%$ y en la Marina el $42 \%$, siendo mucho más bajo en la Gendarmería, con solamente el $93 \%$. Esta tasa de notoriedad puede considerarse satisfactoria habida cuenta de la obsolescencia de las medidas

En cambio, en lo que se refiere a las actuaciones del C.S.F.M. a favor de las ayudas al licenciarse y de las ayudas a la readaptación, puede considerarse bastante bajo en general, en los tres Ejércitos: el porcentaje de respuestas afirmativas es, por lo general, inferior al 50\%, a excepción de la Marina en la que el porcentaje es bastante elevado.

Estas ayudas (nuevas asignaciones, bajas por reconversión) son recientes y fueron objeto de numerosísimas comunicaciones.

En lo concerniente a las actuaciones del C.S.F.M. a favor del $5^{\circ}$ nivel, su tasa de notoriedad es baja. Lo cual es normal ya que afecta a una pequeña parte de los efectivos militares.

- La eficacia del sistema de concertación en el Ejército.

Se evaluó de dos formas diferentes entre los militares:

- de forma indirecta, preguntándoles si los comunicados de las instancias:

a) reflejan las preocupaciones de los militares;

b) tienen bien en cuenta los diferentes aspectos de la condición militar;

c) les siguen actuaciones concretas.

- de forma directa, formulando la pregunta de la eficacia de las instancias.

- La cuasi totalidad de los militares encuestados (91\%) consideran que los comunicados del C.S.F.M. reflejan total o parcialmente sus preocupaciones.

Alrededor del $86 \%$ de los militares tienen la impresión de que a los comunicados del C.S.F.M. les siguen actuaciones concretas.

Para el $83 \%$ de los militares la concertación permite mejorar la condición militar. Es ésta una conclusión que refleja la relevante imagen positiva de que gozan el C.S.F.M. y el C.F.M. entre los militares.

En lo concerniente a la opinión final sobre la eficacia de las respuestas, el nivel de satisfacción decrece con el «orden jerárquico» de las instancias: el 69\% juzgan eficaz al C.S.F.M., el $60 \%$ son de la misma opinión respecto a los C.F.M. Las instancias locales son consideradas menos efectivas ( $44 \%$ de juicios favorables para las comisiones participativas, $52 \%$ para los presidentes de las diferentes categorías). 


\section{Conclusión}

Las estructuras nacionales satisfacen de manera global las expectativas de los militares. Sus notificaciones, sus acciones y su eficacia gozan de una opinión favorable, a pesar de un conocimiento real insuficiente.

Las opiniones, no obstante, varían en función del estatuto (los militares de carrera, mejor informados, tienen una opinión sensiblemente mejor), de la categoría jerárquica y de la antigüedad en el servicio. A señalar el que estas tres características están altamente correlacionadas.

Tercero.- Una comunicación insatisfactoria y una concertación local menos apreciada.

- Una comunicación juzgada insuficiente.

Los vectores privilegiados para la difusión de los trabajos de las instancias de concertación son:

- los informes de las instancias.

- la prensa militar.

La mitad de los oficiales leen los informes de las instancias y cerca del $40 \%$ leen la prensa militar. Al contrario, el 7\% de los soldados leen los informes de las instancias y menos del $30 \%$ leen la prensa militar.

Por lo general, menos de un militar de cada dos acceden a los vectores de comunicación que difunden la información sobre la concertación. Entre ellos, el «nexo» no se conoce bien, lo cual explica, en parte, el desfase entre la notoriedad de las instancias nacionales de concertación y el conocimiento real de dichas instancias.

Esta falta de acceso a la información suscita, a cambio, una fuerte demanda: el $60 \%$ de las 500 personas que formularon comentarios libres reclaman una mejor difusión de la información.

De manera general, la mitad de los militares preguntados estiman que la libertad de expresión de los miembros del C.F.M. y del C.S.F.M. está garantizada totalmente (16\%) o parcialmente. Unicamente el $25 \%$ de los militares consideran que la libertad de expresión es insuficiente. Este último porcentaje es claramente interior entre aquéllos que participaron directamente en las instancias de concertación. Al contrario, los militares que se creen los peor informados, son los más desconfiados respecto a las instancias de concertación, en lo que se refiere a la libertad de expresión o al clima de confianza.

- Las instancias locales

La tasa de notoriedad es muy elevada (95\%), incluso entre la tropa (89\%). Pero únicamente la mitad (53\%) de los militares consideran estas instancias eficaces.

El hecho de que las instancias locales resuelvan problemas cotidianos (funcionamiento, abastecimiento) poco espectaculares en cuanto a su trascendencia, al contrario que las cuestiones tratadas por las instancias nacionales, puede ser la 
causa de esta deficiencia de apreciación global.

En lo que se refiere a estas mismas instancias locales, hay que señalar que el personal de tropa es menos negativo que el resto de las categorías jerárquicas: para ellos las instancias de concertación están relativamente bien valoradas ya que les son más familiares, por lo que su carácter concreto se aprecia con mayor nitidez.

- Conclusión.

Más que una conclusión, una observación: la implicación en el ejército es diferente según se trate de un militar (de carrera) o de un soldado de reemplazo (la mayoría de las veces con contrato). Las preocupaciones son diferentes. Para los primeros poseen una dimensión institucional siendo mucho más locales y concretas para los segundos. Asimismo, poseen una dimensión temporal diferente: los soldados reenganchados con contrato piensan a corto plazo, los militares de carrera tienen una visión más prospectiva.

Estas diferencias pueden explicar el hecho de que los militares de carrera se sientan más implicados en las instancias nacionales que abordan temas más amplios de la condición militar, mientras que los militares con contrato tienen marcado interés por las instancias locales.

Cuarto. - Las expectativas y las propuestas de los militares.

El análisis de los comentarios libres

Además de las preguntas cerradas para las cuales las modalidades de respuesta ya estaban fijadas, el cuestionario propuesto a los militares contenía algunas preguntas abiertas que permitían expresarse libremente y con mayor precisión sobre determinados aspectos de la concertación.

De los 1.748 cuestionarios devueltos al Observatorio, 545 militares utilizaron este tipo de pregunta. La mayoría (más de 300) hicieron hincapié en la mejora de la difusión de la información y, de manera general, de la comunicación. Insistieron, asimismo, sobre la mejora de las condiciones de actuación en el cargo de los miembros que participan en las instancias de concertación.

- Desarrollar la comunicación.

Una comunicación más accesible: podrían aprovecharse ciertos acontecimientos relevantes de la vida militar para difundir la información. Los militares sugieren principalmente la organización de reuniones, cursos o también instrucciones colectivas.

Así mismo, debe realizarse un esfuerzo para que la información cuando sea escrita, sea concisa y menos compleja.

Se hace hincapié sobre una acción pedagógica: la falta de interés y de implicación de los jóvenes podría reducirse mediante una acción, de iniciación á los procedimientos y al estatuto de la concertación en el seno del Ministerio de Defensa en el marco de los diversos cursos a que asisten los militares.

- Valorar el papel de los actores y de las instancias locales.

Surge una petición: la de dar a los participantes en las instancias de concertación 
una mayor formación y disponibilidad. Se manifiesta principalmente la voluntad de que la actividad de los participantes no constituya un freno para su carrera.

Quinto.- Conclusiones generales.

El sistema de concertación local parece satisfacer a la mayoría de los militares.

El C.S.F.M. y el C.F.M. gozan de una imagen de eficacia.

Estas instancias responden globalmente a las preocupaciones de los militares.

Sin embargo,

Las instancias locales sufren una pérdida de imagen.

El papel y las actuaciones de las instancias de concertación sólo se conocen, a veces, de forma ambigua. Ahora bien, un conocimiento deficiente de estas últimas altera el clima de confianza. La dirección principal de mejora pasa, por tanto, por la potenciación de la comunicación.

Pierre Azoulay 1999.

$* * *$

\section{Cuarto. Libertad de expresión: uso y práctica.}

Coronel de Richoufftz ${ }^{6}$, Ejército de Tierra, Francia.

Soy coronel del ejército de tierra francés y más allá de una vida operativa especialmente fructífera y de una de experiencia militar extraordinaria en muchos campos, podría considerárseme una excepción en este final de siglo, al menos dentro de la institución a la que hoy represento aquí. En efecto, soy el único oficial del Ejército francés, en activo y que viste uniforme, que haya escrito bajo su identidad y en diez años, tres obras. Debo decir que tal excepción, a veces dura de soportar y que por supuesto influyó en mi carrera, tiene algo de enriquecedor. $Y$, porqué no confesarlo, salir del anonimato, de un conformismo placentero gracias a las publicaciones, no es algo que me disguste. No contentarse con el orden establecido, el afán por ir a la par con la evolución política y estratégica, no olvidar que el soldado debe seguir siendo la principal de nuestras preocupaciones, me llevaron pues a escribir. En razón a un cierto gusto por el riesgo y brabuconería frente a una jerarquía, inicialmente timorata para con los autores militares y actualmente, condescendiente o, más exactamente, no opuesta a que los militares de uniforme se expresen, hice uso de la pluma a fin de decir lo que pienso. Por esto mismo, creo que describo, de alguna manera, lo que más o menos siente una categoría social que casi nunca se expresa. No es mi intención relatarles los rodeos administrativos que hay que dar antes de obtener autorización para hablar o escribir. Otros que conocen los textos a la perfección podrán hacerlo mucho mejor que yo. No; simplemente quisiera compartir con ustedes todas mis 
convicciones - ¿por qué escribir? - y reticencias, numerosas en la senda de la escritura, que hay que superar una vez que uno ha creado su obra. Por último, no sería ecuánime si no mencionase la cuestión fundamental que se plantea todo autor: convencer. En efecto, escribir no es mi actividad principal y no son los cientos de páginas emborronadas durante meses, noches, fines de semana y vacaciones, las que me permitirían alimentar a una familia... Una cantidad módica sujeta, por lo demás, a una base imponible. No; creo que escribir responde a otro objetivo, al menos en mí caso: hacer que los militares tomen conciencia de los grandes retos y hacer evolucionar el pensamiento militar. Así, les propongo seguir el siguiente esquema, dividiéndolo en tres partes: porqué escribir, vencer, convencer. También les pido que tengan en mente el considerable cambio de mentalidad que se produjo en estos últimos diez años: de una hostilidad reprobadora a una aceptación real. Es una importante evolución para nuestra institución, a la que no soy ajeno, creo yo.

$1^{\circ}$ ¿Por qué escribir?

Para mí fue una cuestión que nunca se planteó. Pienso que mi vida de oficial quedaría incompleta si no me hubiera expresado en los momentos más delicados que afectaron a nuestra vida. Adecuar mis actos a mi conciencia. En estas condiciones, el discurso ya no basta. Las palabras se las lleva el viento: hay que escribir. Para mí, cada libro surgió de un «clic». Cada uno de ellos supuso la ocasión para una toma de conciencia, toma de conciencia que lleva a una reflexión esencial.

1988: ¿para qué sirve lo nuclear frente a las nuevas formas de guerra (terrorismo urbano)? Y proponer una reducción drástica de la panoplia militar y privilegiar el entrenamiento de las unidades del ejército de tierra en medio urbano.

1992: ¿para qué sirve un ejército de reclutamiento desde el momento en que los intereses vitales del país no se ven amenazados (Guerra del Golfo)? $Y$ prever, entre otras cosas, un Ejército profesional reducido en cuanto a efectivos y proyectable fuera de nuestras fronteras nacionales.

1998: ¿en nombre de quién o de qué puede pedirse a un soldado empeñado en una coalición que dé su vida (OTAN, ONU...)? Y redefinir o definir nuevos ideales que puedan transcender a la idea de nación.

Así, desde mi punto de vista, escribir responde realmente a una iniciativa seria que deseo compartir con aquéllas y aquéllos que experimenten, en un momento dado de su vida de soldado, la necesidad de preguntarse a sí mismos.

$Y$ esta consulta intrínseca es tanto más necesaria cuanto que nuestro mundo evoluciona a pasos gigantescos y que ningún ámbito de nuestra vida parece no poder sustraerse a dicho movimiento. En tales condiciones, no cuestionarse a uno mismo, en estos momentos, me parece un error, una negligencia. No olvidemos jamás que nuestro primer deber es mandar a hombres en situaciones bélicas, prepararlos para la guerra. No tener nunca que ocultar la verdad. A los soldados no se les miente. Éstos deben saber lo que se espera de ellos. El sacrificio último 
bien merece una respuesta. En estas condiciones la intervención no puede realizarse a partir de un pensamiento único. Y el ciudadano que soy, que ejerce su derecho de voto, también tiene, por tanto, el derecho a elegir; ¿por qué razón tendría que verse privado entonces del derecho de expresión? ¿No es éste un derecho fundamental reconocido en nuestras democracias?

Entiéndase muy bien que mi actuación solitaria —soledad que a veces resulta muy dificil de asumir - se inscribe en un campo muy distinto al de los historiadores militares, quienes relatan hechos de armas y días gloriosos de prestigiosas unidades con las que generalmente intervinieron en un momento crucial de nuestra historia.

Aún a riesgo de desilusionar, no puedo pasar por alto las numerosas publicaciones de militares en la reserva que a veces se expresan en los titulares de los periódicos parisinos, pero con menos frecuencia a través de libros de consulta. ¿Qué crédito otorgar a aquéllos que fueron los jefes de nuestros ejércitos en el pasado y que, en activo, jamás tomaron una decisión: escribir con toda libertad? ¿Se trata de una revancha, tardía, de una cierta falta de valor o de un ocio mal llevado? En cualquier caso son acciones no necesariamente bien acogidas por la institución. Una pregunta quema la lengua: ¿cuántos, de estos escritores retirados, podrían enorgullecerse de ser oficiales generales si hubieran tenido el valor de coger la pluma cuando sólo eran tenientes-coroneles o coroneles? No muchos, ciertamente...

En cuanto a mí, vestir el uniforme es comprometerse, en el sentido noble del término. Aunque para ello sea preciso saber vencer las reticencias, y otros muchas contingencias que ahogan la libertad de expresión.

2. Vencer las reticencias.

No crean que porque hayan finalizado su manuscrito éste vaya necesariamente a publicarse (...) tras meses de intensa labor nocturna y el orgullo de disponer de una primera prueba que responde a su compromiso inicial: desgraciadamente esto no basta. Hay que recorrer un trayecto sinuoso para el que se requiere poseer las cualidades de un corredor de fondo: vencer las reticencias cualesquiera que sea su procedencia y que surgen diaria y repetidamente. Las resistencias de la propia institución, la incredulidad del editor y las reticencias por parte del lector. Una lucha que tuve que librar y repetir con cada título.

- La institución militar es, por esencia, conservadora y recelosa desde el momento en que a uno de sus miembros se le ocurre emitir una opinión la cual, siempre tomada en primer grado, se asimila a una crítica. No se critica a la institución cuando se pertenece a ella. Como les decía en la introducción, eso fue lo que experimenté en 1987-1988. Vencer la desconfianza a toda costa y no correr la misma suerte que unos cuantos oficiales superiores los cuales, entre 1975 y 1982, se vieron obligados a dimitir por haberse atrevido a publicar. Por ello tuve que soslayar el obstáculo valiéndome de astucias. Fijar al adversario y después envolverlo; ¿no es ésta la táctica que se enseña en nuestras academias militares? Pedir autorización es seguir el reglamento: con ello se fija la atención de 
las autoridades. Al mismo tiempo, e incluso anticipándome ligeramente al proceso, procedía a rebasar sistemáticamente al adversario: visitar a las autoridades a fin de explicarles el contenido de la obra. De entrada, sortear la crítica a fin de obtener la autorización previa; ya que sin esta última, imposible publicar salvo que uno quiera hacerse el harakiri.

Permitanme que les cuente como pude llevar a cabo mi decisión:

en 1987, mediante un "efecto de sorpresa estival",

en 1992, mediante una ofensiva «sin idea de retroceso»,

en 1998, mediante el «envolvimiento de la posición».

(Podría abundar en este punto a la hora de las preguntas)

Si la autorización para publicar es condición sine qua non, aceptar la idea de que un oficial pueda expresarse, es algo no necesariamente compartido por la jerarquía. Sin duda alguna, en diez años, las cosas evolucionaron mucho. Pero hay que superar un importante obstáculo: el editor.

Un editor, cualquiera que sea su popularidad, no actúa por filantropía. Como empresario, gana dinero. La competencia es severa en el mundo editorial; no menos de 40 nuevos títulos salen a la luz diariamente en las librerías francesas. En estas condiciones cómo esperar que a uno le editen sus obras siendo un desconocido en el mundo de la edición y que la obra que uno propone no entra en la línea de los «best-sellers». Eso equivale a decir que nuestras posibilidades son escasas cuando proponemos un tema tan poco contagioso como el de defensa.

Debo decir que, en mi caso, he tenido suerte hasta ahora. Único en mi especie en el mercado, por decirlo así, poseía no obstante algunas "características» atractivas a los ojos del editor: en activo, un título llamativo, una publicidad un poco a bombo y platillos. Evitaba, no obstante, que el editor me hiciese pasar por mártir o por un valiente caballero guerreando contra una institución militar intransigente. ;Son tantos y tantos escollos los que hay que eliminar!. En efecto, ¿qué credibilidad podría ofrecer a mis compañeros si me dejase engañar por una publicidad desmedida? Sin embargo, hay que ser conscientes de que el editor, cuyo único objetivo es vender, está buscando siempre una cierta provocación. Mí lucha, como ya saben, es otra.

Son, por tanto. dos líricas antagónicas y en calidad de militar y oficial uno está, permanentemente, en el filo de la navaja. Pero, ¿se da cuenta de ello el lector?

El lector representa, para el autor, el blanco a alcanzar. El objetivo a conseguir mediante la ofensiva de las palabras. La voluntad de vencer por la fuerza de la convicción. Yo, en mi optimismo, había pensado que el prototipo de lector sería un militar.

En efecto, ¿quién podría imaginarse no encontrar en el seno de nuestras Fuerzas Armadas -Tierra, Mar, Aire y Gendarmería -, de la Delegación General de Armamento (DGA) y, por qué no, en el seno de la Policía Nacional, un potencial electorado? Mi decepción fue mayúscula, pero me acostumbré a ello, al constatar 
que únicamente una ínfima minoría (junos cuantos cientos de un total de 500 000!) se toma la molestia de leer. Por temor a la jerarquía (lo he comprobado), por envidia (sin duda), por desinterés del tema militar (muy ciertamente, por desgracia), por temor a tener que gastarse unas perrillas (si). Todos estos elementos se aúnan haciendo primero que el autor militar sea criticado por aquéllos que incluso no lo leyeron, después progresivamente marginado dentro de la institución y, finalmente, empujado a abandonar definitivamente la partida. Pero tranquilicense ustedes, el infante es tenaz cuando emprende una acción. La tercera tentativa fue la buena. Las reticencias parecen finalmente haber sido superadas dentro de la institución. Pero hay que potenciar a toda costa un segundo frente. Lo han adivinado: se trata de difundir el mensaje al mayor número posible de gente, sin lo cual escribir carecería de sentido. Este segundo frente galvaniza las energías: se trata de convencer.

\section{Convencer.}

Así, revestido de la autorización ministerial, y después de haber conseguido, no sin grandes dificultades, un editor, el combate no puede darse aún por ganado. Desde ahora habrá que abrirse paso a codazos a fin de hacernos oír en la sociedad. Nada hay más difícil. Eso es tanto como decir que la empresa está abocada al fracaso si uno trata de desafiar, solo, la vida diaria de aquéllas y aquéllos que tienen bastantes más preocupaciones que el escuchar o leer a uno. Sin la ayuda de los medios de comunicación, apenas hay esperanzas de salir del anonimato. Nos vemos, entonces, obligados a "vender a domicilio» entre las instancias mediatices, a encontrar la ayuda adecuada para que la obra pueda figurar entre las diferentes publicaciones o a «llamar la atención» con emisiones dirigidas al público en general. Es un combate sin tregua, agotador, decepcionante en muchas ocasiones, pero necesario.

- La intervención ante la prensa militar es indispensable: darse a conocer dentro de la institución. Nada más complejo, de hecho. Aceptado a regañadientes, uno se convierte, no obstante, en alguien poco recomendable ya que las ideas que uno desarrolla no son, necesariamente, compartidas por la jerarquía. Así, las múltiples publicaciones militares evitan mencionar la obra. ¿Una censura velada'? Sin duda, doblemente. Pero, ¿no supone, por el contrario, un inconveniente hablar de uno mismo? ¿No supone dar crédito a la falsa idea de que el coronel de Richoufftz se ha convertido en el portavoz de la jerarquía en el seno de la institución militar? Difícil de admitir, como pueden muy bien comprender. Serlo todo a la vez, dentro y fuera. Tanto más cuanto que el público militar compra poco, e incluso nada como decía anteriormente. Entonces, ¿qué esperar de la prensa militar? Una divulgación de nuestra identidad a falta de un reconocimiento. Ya que, ser conocido, para bien o para mal, es una garantía para el futuro: evitar ser marginado de forma definitiva.

- La intervención ante los medios de comunicación nacionales es aún más 
compleja, ya que las segundas intenciones nunca faltan en las relaciones con tal o cual periodista. Nuestras preocupaciones son muy diferentes. Para mí, se trata de transmitir mis ideas al público en general inscribiendo al mismo tiempo mi gestión en un estricto deber de reserva. Para los periodistas, se trata de ponerme la zancadilla a fin de tener una "primicia» en términos de audiencia y lectores. En este campo igualmente la senda es estrecha y la competencia dura. La obra interesa cuando la actualidad es candente: en este caso su intervención es deseada sin ningún tipo de miramientos. Es el acontecimiento el que es el «rey». Para el autor, por el contrario, lo más importante es la reflexión; y ésta se inscribe en el tiempo, fuera de los acontecimientos. Lo esencial sigue siendo la reflexión. Dos preocupaciones diferentes. Sin embargo. Los medios de comunicación siempre «jugaron limpio» conmigo y las críticas han sido más bien halagüeñas. Sin duda fue una recompensa a la tenacidad. La contrapartida no estriba en unos cuantos lectores de más atraídos por las zalamerías y provocaciones de artículos en periódicos conocidos. Es, ante todo, la cuasi certeza de estar poco más o menos a salvo de la jerarquía. ¿Cómo tomarla con un oficial del que hablan los medios de comunicación? Así, pude disponer de una libertad de acción que me permitió consagrarme por entero al público.

- La intervención ante el público es esencial ya que dependiendo de como uno defienda sus ideas, de la acogida dispensada, el público estará o no estará convencido de nuestra gestión. Se adherirá o no se adherirá a las ideas que uno defiende. Es una intervención normal a llevar a cabo con «blancos» diferentes, pero todos ellos con una característica común: convertirse, para los demás, en el embajador convencido de lo equitativo de sus ideas. Dedicatorias en librerías, en grandes superficies o en ferias de libros son un primer paso. Conferencias o debates con una gran afluencia de público civil o militar que permita ampliar el círculo, son un segundo. Debo decir que tuve la fortuna de poder expresarme ante varios auditorios compuestos por hasta 350 personas, lo cual puede considerarse un éxito teniendo en cuenta que algunos políticos franceses sólo llegan a un diez por ciento de público. La conjugación de medios de comunicación/expresión en público me parece una buena fórmula para convencer. Diálogo directo escalonado en el tiempo junto con un soporte mediático que preceda la intervención en publico y pueda dar cuenta de los debates. Es un trabajo de larga duración que, como ya saben, requiere una intensa y fatigosa dedicación personal, una organización que no permite el poco-más-o-menos, sino la satisfacción de llegar hasta el final de lo que uno se ha propuesto. Ésa fue la sensación que experimenté durante estos cuatro últimos meses. Y cuando academias, organismos, empresas, universidades desean recibir a uno - la prueba es que están ustedes hoy aquí-, supone un cierto reconocimiento y, quien sabe, un preludio de notoriedad. Se ha dado en el clavo. Pero convencer es un esfuerzo constante que exige abnegación y pasión. Espero haberles convencido tanto de la legitimidad de la expresión de los militares 
en el seno de una institución que, como otras muchas, sufre importantes convulsiones, como del combate en solitario que el autor que viste uniforme se ve obligado a librar. No es algo fácil, repito, pero es fascinante. Se lo aseguro: tampoco en este campo hay victorias fáciles.

Así, llego al final de mi exposición. Espero haberles convencido de que si la libertad de expresión existe en el seno de nuestra institución en Francia, el autor se halla casi siempre solo a la hora de defender sus ideas. Es, a veces, dificil de llevar y el resultado, incierto. Es lo que he tratado de decirles durante todo este tiempo. No obstante, tengo el sentimiento de que mi humilde experiencia, durante estos diez años, sirvió para la libertad de expresión.

Modestamente, creo estar en condiciones de poder decir que contribuí a entreabrir la puerta de lo escrito, de otra forma de hablar. Ahora le toca el turno a otros. En un periodo especialmente fructífero, hay mucho qué hacer y qué decir. Tengo esperanzas en que me salgan muchos émulos; ;ánimo!. Suerte. Gracias por su atención. Cor. Richoufftz, 1999. 


\section{NOTAS}

${ }^{1}$ Más exactamente, este autor los cifra de la siguiente forma: «(...) los tres problemas prioritarios fueron: la sociología del conflicto político militar; la sociología del militar de carrera, y la sociología de las reformas militares», ALONSO BAQUER, M., «La sociología militar en España», en: HARRIES-JENKINS, G. y MOSCOS Jr., Ch.C., (1984): Las fuerzas armadas y la sociedad, Alianza, Madrid, p.25. Esta obra es una pieza de referencia básica en el campo de la sociología militar.

2 Transcripción y traducción de la grabación de la intervención del Interventor General del Ejército francés Rebsmeiter,Toledo, 1-2 de Julio de 1999, A.C.M.S., Ministerio de Defensa. Rev. por Grupo de trabajo de Sociología Militar de la ACMS. ${ }^{3}$ Falta en este punto una breve porción de la intervención que no figuraba en la documentación de origen. (N. del editor).

${ }^{4}$ (sic.), «Langue de bois». N.del T.

${ }^{5}$ Traducción realizada por el Gabinete de Traductores e Intérpretes de la SEGENEME, registrada con el n ${ }^{\circ} 52 \mathrm{~S}-99$.

${ }^{6}$ Bibliografía del coronel Emmanuel de Richoufftz: —Décembre 1997, les Russes arrivent: un officier d'active raconte, A. Michel, París, 1987, (ISBN: 2-226-03196-0); -Encore une guerre en retard?: Un officier d'active ose parler, A.Michel, París, 1992, (ISBN: 2-226-05717-X); - Pour qui meurt-on?, A.D.D.I.M., col. Esprit de défense, Paris, 1998, (ISBN: 2-907341-87-1). (N.del editor). 